\title{
Psoriasis and infection. A clinical practice narrative.
}

Rademaker M, Agnew K, Anagnostou N, Andrews M, Armour K, Baker C, Foley P,

Gebauer K, Gupta M, Marshman G, Rubel D, Sullivan J, Wong L-C.

Address for correspondence: Hon. Associate Professor Marius Rademaker, Waikato Clinical School, Auckland University Medical School, Hamilton, New Zealand

Tel: +6478381035

Email: Rademaker@xtra.co.nz

- Word Count: 3767

- Figures: 0

- Tables: 0

Running head: Psoriasis and infection

Key words: psoriasis, infection, vaccine, therapy, surgery, wound healing

Acknowledgments: We are very grateful to AbbVie Pty Ltd (Australia) for an unrestricted educational grant. They had no influence in the subject matter, discussion or writing up. We are grateful for Tony James, independent medical writer for taking notes during the meeting. He was not otherwise involved in the preparation of this manuscript.

\section{Authorship:}

- Design, literature search, discussion, conclusions, final version of manuscript all authors

- Manuscript preparation - MR

\section{Conflicts of Interest:}

This is the author manuscript accepted for publication and has undergone full peer review but has not been through the copyediting, typesetting, pagination and proofreading process, which may lead to differences between this version and the Version of Record. Please cite this article as doi: 10.1111/ajd.12895

This article is protected by copyright. All rights reserved 


\begin{tabular}{|l|l|}
\hline Rademaker & Clinical investigator: AbbVie, Douglas Pharmaceuticals. \\
\hline Agnew & none \\
\hline Anagnostou & none \\
\hline Andrews & none \\
\hline Armour & Speaker and/or Advisory Board: AbbVie, Janssen-Cilag, Novartis, Sun \\
Pharma.
\end{tabular}

Name/Degree/Affiliation:

\begin{tabular}{|l|l|l|}
\hline Name & $\begin{array}{l}\text { Highest } \\
\text { Degree }\end{array}$ & Affiliation \\
\hline Rademaker & DM & $\begin{array}{l}\text { Waikato Clinical School, Auckland University Medical } \\
\text { School, Hamilton, New Zealand }\end{array}$ \\
\hline Agnew & FRACP & $\begin{array}{l}\text { Dermatology Department, Greenlane Clinical Centre \& } \\
\text { Starship Children's Hospital, Auckland, New Zealand }\end{array}$ \\
\hline Anagnostou & FRACP & Southern Adelaide Local Health Network, SA, Australia \\
\hline
\end{tabular}

This article is protected by copyright. All rights reserved 


\begin{tabular}{|l|l|l|}
\hline Andrews & FACD & 68 Ipswich Rd, Woolloongabba QLD, Australia \\
\hline Armour & FACD & Skin \& Cancer Foundation Inc., Melbourne, VIC, Australia \\
\hline Baker & FACD & $\begin{array}{l}\text { The University of Melbourne, St Vincent's Hospital, } \\
\text { Melbourne; and Skin \& Cancer Foundation Inc., } \\
\text { Melbourne, VIC, Australia }\end{array}$ \\
\hline Foley & FACD & $\begin{array}{l}\text { The University of Melbourne, St Vincent's Hospital, } \\
\text { Melbourne; and Skin \& Cancer Foundation Inc., } \\
\text { Melbourne, VIC, Australia }\end{array}$ \\
\hline Gebauer & FACD & $\begin{array}{l}\text { University of Western Australia, Perth; and Probity } \\
\text { Medical Research, Freemantle, WA, Australia }\end{array}$ \\
\hline Gupta & FACD & $\begin{array}{l}\text { Department of Dermatology, Liverpool Hospital, Sydney; } \\
\text { The Skin Hospital, Darlinghurst; and University of New } \\
\text { South Wales, Sydney, NSW, Australia }\end{array}$ \\
\hline Marshman & FACD & $\begin{array}{l}\text { Flinders Medical Centre; and Flinders University Medical } \\
\text { School, Adelaide, SA, Australia }\end{array}$ \\
\hline Rubel & FACD & $\begin{array}{l}\text { Woden Dermatology, Phillip, ACT; and Australian National } \\
\text { University, ACT, Australia }\end{array}$ \\
\hline Wong & FACD & $\begin{array}{l}\text { Dermatology Department, The Children's Hospital at } \\
\text { Westmead, NSW, Australia }\end{array}$ \\
\hline Kingsway Dermatology, Miranda NSW, Australia
\end{tabular}

This article is protected by copyright. All rights reserved 
PROF. MARIUS RADEMAKER (Orcid ID : 0000-0003-3393-6748)

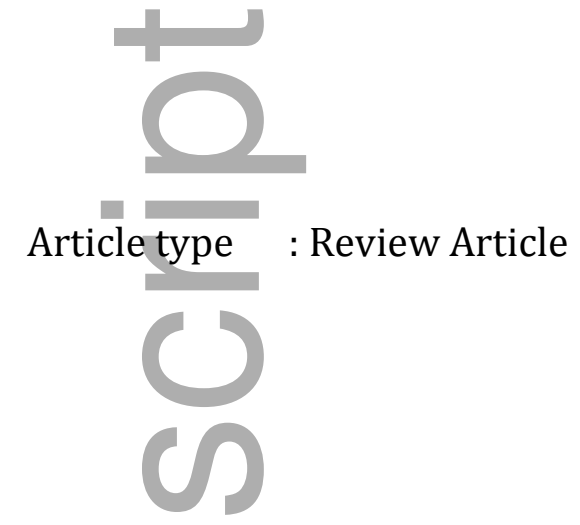

\section{Psoriasis and infection. A Clinical Practice narrative.}

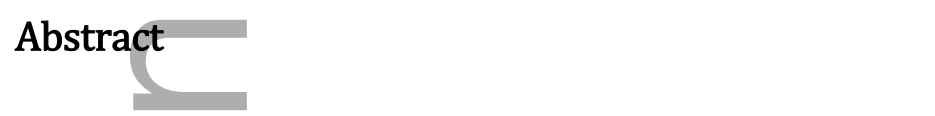

The Australasian Psoriasis Collaboration has developed a clinical practice narrative with respect to the relationship between psoriasis, its treatment and infection. The cutaneous microbiome of patients with psoriasis is different to those without psoriasis, although the significance of this is unclear. Whilst a wide-range of microorganisms have been associated with psoriasis (including $\beta$-haemolytic streptococci, Staphylococcus aureus, Porphyromonas gingivalis, Candida albicans, Chlamydia psittaci, Human Immunodeficiency virus and Hepatitis C virus), there is limited evidence that antimicrobial therapy is of direct benefit in preventing flares of psoriasis.

Psoriasis is independently associated with an increased risk of serious infection, but the absolute risk is low. The risk of serious infections is further increased with immune-modulatory treatments. The decision whether to, and when to, stop or resume immune-modulatory treatment after a serious infection has occurred depends on risk assessment for that patient, taking into account the infection being treated, the risk of recurrent infection, any interventions that can modify the risk, and the need for psoriasis control. 
Live vaccines (e.g. MMR, varicella, zoster, yellow fever) are generally contraindicated in patients with psoriasis on immune-modulatory agents, but this depends on the degree of immune suppression and individual risk factors.

Wound healing in psoriasis is normal. Treatment with infliximab, adalimumab, etanercept, methotrexate and ciclosporin can safely be continued through low-risk surgical procedures. For moderate- and high-risk surgeries, a case-by-case approach should be taken based on the patient's individual risk factors and comorbidities.

\section{Introduction}

The advent of therapies that target specific components of the immune response have highlighted the possible associations of infection with psoriasis. The Australasian Psoriasis Collaboration ${ }^{1,2}$ held a round table discussion to develop a clinical practice narrative with respect to:

- infections that may trigger psoriasis

- infections that may occur in patients with psoriasis

- infections in association with specific treatment of psoriasis

- the treatment of infections in patients with psoriasis and on specific antipsoriatic therapies

- strategies to reduce infection in patients with psoriasis, including immunisation and travel advice.

- surgery, wound care and healing in psoriasis

The following narrative is divided into 4 sections, and makes a number of clinical observations and recommendations, based on the published literature and expert clinical opinion. Much of the available evidence is of low quality consisting of case reports, case series and cohort studies. The quality of evidence from randomised clinical trials of biologic therapies for psoriasis is better, but is mostly indirect with regards to the topics covered.

\section{Infections that may trigger psoriasis}

This article is protected by copyright. All rights reserved 
The cutaneous microbiome of patients with psoriasis is different from those without psoriasis.3,4 The three most common phyla in both the general population and psoriasis skin are Firmicutes, which constitutes up to 39\% of the microbiota in psoriasis, compared to $43 \%$ of normal skin, Proteobacteria (38\% psoriasis, $27 \%$ normal skin) and Actinobacteria (5\% psoriasis, $16 \%$ normal skin) (p=0.034). The commonest genus of skin bacteria is Streptococcus, in both psoriasis (32\%) and normal skin (26\%). Staphylococci are less common in psoriasis (5\%) than in controls (16\%), as are Propionibacteria (psoriasis 0.0001\%, controls $0.025 \%$ ). Although the significance of these differences in the microbiome are unclear, it has been proposed that a breakdown of immune tolerance from disordered microbiota of the skin ${ }^{5}$ may contribute to the onset of psoriasis, similar to a current hypothesis of the role of intestinal microbiota and Crohn disease. ${ }^{6-7}$ However, the extent of the changes in the microbiome do not seem to correlate with the severity of psoriasis. Interestingly, patients with Crohn disease have a fivefold higher risk of developing psoriasis than those without, whilst patients with psoriasis are also more likely to suffer from Crohn disease. ${ }^{5}$

\section{Streptococci}

Throat infection with $\beta$-haemolytic streptococci has long been associated with both the initiation and acute exacerbation of psoriasis. ${ }^{8-10}$ In one study, patients with psoriasis reported a sore throat 10 times more often than household controls. ${ }^{9}$ The mechanism of triggering psoriasis is thought to be via bacterial superantigens able to stimulate $\mathrm{T}$ cell proliferation without prior intracellular processing by an antigenpresenting cell. ${ }^{11}$ Infiltrating T cells, which can drive psoriatic skin disease, might originate in tonsils where streptococcal infection induces a skin-homing phenotype in which streptococcal and skin-specific epitopes interact via molecular mimicry. This is likely to be partly genetic as $100 \%$ of 29 patients with guttate psoriasis carried the $\mathrm{Cw}^{*} 0602$ allele compared to only $20 \%$ of 604 controls. ${ }^{12}$

Despite the large number of case reports and series, a 2001 systematic review ${ }^{11}$ found no good evidence of benefit from the use of antibiotics in patients with acute 
guttate psoriasis or chronic plaque psoriasis, mainly due to the absence of satisfactory studies comparing antibiotic therapy with placebo. There are a number of case series ${ }^{9}$ that recommend tonsillectomy for selected patients with guttate and pustular psoriasis, but there are no controlled studies to confirm a benefit. It is possible that tonsillectomy, but not antibiotic therapy, removes offending antigen thereby assisting in disease control. ${ }^{13}$

In addition to the association between streptotococcal infections of the throat and psoriasis, there are a number of case reports of guttate psoriasis in association with perianal streptococcal disease in children ${ }^{14}$ as well as streptotococcal infections and pustulosis palmaris et plantaris/localised pustular psoriasis. ${ }^{15}$

\section{Comments/Learning points 1 :}

- The cutaneous microbiome of patients with psoriasis is different from those without psoriasis, but the significance of this is unclear.

- Several organisms including $\beta$-haemolytic streptococci, Candida albicans and Hepatitis $C$ virus have been shown to trigger psoriasis.

- There is no good evidence that systemic antibiotics reduce streptococcal sore throat triggered flares of guttate psoriasis. Tonsillectomy may however, be effective.

- There is limited value in indiscriminate screening for infection in patients with newly diagnosed psoriasis.

\section{$\underline{\text { Staphylococci }}$}

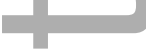

A systematic review of staphylococcal colonisation in psoriasis identified 21 studies; ${ }^{16}$ patients with psoriasis were 4.5 -times more likely to be colonised by $S$. aureus than healthy controls [relative risk (RR) and (95\%CI): skin: 5.54 (3.21-9.57); nares: 1.60 (1.11-2.32)], differing from microbiota studies ${ }^{3,4}$ where a lower prevalence of Staphylococcus spp. was found in patients with psoriasis. In a further study, a high proportion of $S$. aureus isolates in persons with psoriasis secreted staphylococcal enterotoxins; interestingly, those patients had a significantly higher 
Psoriasis Area and Severity Index (PASI) score compared to those with toxin negative $S$. aureus isolates. ${ }^{17}$

\section{Other bacteria}

Other organisms linked to psoriasis include colonic bacteria Enterococcus faecalis, Escherichia coli, and Pseudomonas aeruginosa and Proteus species but the evidence is weak. ${ }^{18,19}$ One study of 300 patients with psoriasis, and 150 healthy controls without psoriasis, found an association between infection with Helicobacter pylori and disease severity, and showed that eradicating $H$. pylori enhanced the effectiveness of psoriasis treatment. ${ }^{20}$

The evidence for bacterial periodontitis being associated with psoriasis is stronger. A systematic review found a pooled risk ratio of 1.55 (95\% CI, 1.35-1.77) for psoriasis in patients with periodontitis. ${ }^{21}$ The link is thought possibly to be Porphyromonas gingivalis, a common Gram-negative anaerobic periodontal pathogen.

Further evidence of the role of bacterial infection triggering psoriasis comes from a nested case control study, ${ }^{22}$ which demonstrated infections were associated with the development of paediatric psoriasis [adjusted odds ratio (aOR) and (95\%CI), 1.2 (1.0-1.5)]. Untreated non-skin infections [aOR, 1.5 (1.3-1.8)], but not antibiotictreated non-skin infections [aOR, $1.1(0.9-1.4)]$, were associated with development of psoriasis.

Subclinical Chlamydia psittaci infection has been found in 17\% (11/64) of individuals with psoriasis versus only $0.4 \%(1 / 225)$ of healthy controls. Chlamydia species behave as obligate intracellular parasites, and are a common cause of inflammatory diseases. C. psittaci positivity is raised in a number of inflammatory diseases including chronic polyarthritis (13\%), psoriatic arthritis (17\%) and seronegative rheumatoid arthritis (28\%). ${ }^{23}$

\section{Fungi/yeasts}


Candida spp. have been isolated in 15\% of skin specimens from patients with psoriasis compared with just $4 \%$ of controls ( $p=0.045$ ), and in $60 \%$ of oral specimens in patients with psoriasis compared with $20 \%$ of controls $(\mathrm{p}<0.01){ }^{24}$ Candida spp. are also found twice as often in the faeces of patients with psoriasis $(68 \%-78 \%)$ compared to controls (29\% - 54\%). ${ }^{25,26}$ Interestingly though, anticandidal antibodies are lower in patients with psoriasis compared with controls, suggesting that psoriasis may be associated with a reduction in humoral immune response to candida. Some Candida spp. secrete toxins that may exacerbate psoriasis, and it has been hypothesised that the presence of $C$. albicans in the gut can stimulate production of superantigens, causing non-specific T-cell activation and production of cytokines that can trigger the psoriatic process. ${ }^{5,24,26,27}$ A study by Buslau et al, demonstrated improvement in more that half of the 50 patients with psoriasis treated with oral nystatin. ${ }^{28}$

Prevalence and species studies of Malassezia spp. in psoriasis vary, but a number of species have been implicated including M. globosa, M. furfur and M. sympodialis (these later two found commonly in scalp lesions of psoriasis). ${ }^{29}$ Mechanistically, Malassezia spp. have been shown to upregulate keratinocyte expression of transforming growth factor- $\beta 1$, heat shock protein 70 , and integrin chain, which were all expressed more in plaques of psoriasis colonised with malassezia, than in non-colonised psoriatic plaques. The scalp is a common site for psoriasis, and several reports have shown improvement following oral ketoconazole, associated with a reduction in yeast numbers. ${ }^{30}$

\section{$\underline{\text { Viruses }}$}

The prevalence of human papilloma virus (HPV) in hair and skin from 20 patients with psoriasis was substantially higher than in 23 controls (83\% vs. 47\%, respectively, $\mathrm{P}<0.03)$. However this may be due to psoriatic plaques being more permissive for viral presence than normal skin. ${ }^{31}$ It will be interesting to see whether wide spread uptake of the new 9-valent HPV vaccine alters the prevalence of adolescent psoriasis.

This article is protected by copyright. All rights reserved 
Retroviruses were first implicated in psoriasis more than 30 years ago. People with psoriasis are reported to have a higher prevalence of circulating IgG antibodies to murine leukemia virus than controls. ${ }^{32}$ Interestingly, psoriasis patients are significantly more likely than controls to have gene variants that are protective against human immunodeficiency virus-1 disease. ${ }^{33}$

Psoriasis may appear for the first time, or pre-existing psoriasis may flare, in patients who acquire human immunodeficiency virus (HIV) infection; ${ }^{34}$ the hazard ratio for incident psoriasis in HIV has been calculated at 1.8. ${ }^{5}$ The paradoxical exacerbation of psoriasis, a T cell-mediated disease, in patients with HIV, in which circulating CD4+ T cells are depleted, has not been fully explained.

The data on Hepatitis B and C (HBV and HCV) is mixed, with one epidemiologic survey finding a higher prevalence of HCV infection, but not HBV infection, in psoriasis. $^{36}$ A study of 717 Japanese patients with psoriasis found that $7.5 \%$ were anti-HCV antibody-positive. ${ }^{37} \mathrm{HCV}$ infection preceded the onset of psoriasis in almost all (definitely - 80\%, probably - 11\%); HCV may upregulate inflammatory cytokines, possibly increasing susceptibility to developing psoriasis. A study by Chun et al demonstrated higher levels of cathelicidin, toll-like receptor (TLR)-9 and interferon (IFN)-c in both non-lesional and lesional skin of HCV-positive patients with psoriasis as compared to HCV-negative individuals with psoriasis. ${ }^{38}$

There is a case series of Chikungunya infection triggering or exacerbating psoriasis in 7 patients. ${ }^{39}$

\section{Infections that may occur in patients with psoriasis, or in association with treatment}

Patients with psoriasis are at increased risk of serious infection. A Dutch cohort study found an increased risk of serious infection [Hazard ratio (HR) and (95\% CI): 1.54 (1.44-1.65)] with the greatest risk in patients with more severe psoriasis; this was independent of treatment (HR, 1.81 (1.57-2.08)]. ${ }^{40}$ Respiratory tract, abdominal, and skin infections were the more common infections among patients with psoriasis. Similarly, a cohort study from Taiwan reported an increased risk of This article is protected by copyright. All rights reserved 
hospitalized pneumonia among patients with psoriasis, independent of other potential risk factors for pneumonia [HR, 1.40 (1.12-1.73)]. Once again, there was a direct relationship with the severity of the psoriasis [HR, 1.68 (1.12-2.52)].41,42

Whilst there is a sound biological rationale for increased risk of infection following treatment with immune-modulators including tumour necrosis factor-alpha (TNF$\alpha$ ) and interleukin (IL)-23/IL-12 inhibitors, the clinical evidence is confounded by these agents being used in more severe psoriasis, and with the co-morbidities of severe psoriasis, including smoking, obesity, diabetes and metabolic syndrome. The PSONET registry ${ }^{43}$ did not demonstrate an increased risk of infection, but other studies have. In the PSOLAR registry, ${ }^{44}$ based on 22,918 cumulative patient-years of follow-up, the unadjusted rates of serious infection based on exposure within 91 days preceding the event were: ustekinumab 0.89 events/100 patient years of observation, infliximab/golimumab (2.86), other biologics (1.71), non-biologics (1.16), and overall 1.44 per 100 patient years. The risk of serious infection independently increased with age, comorbidities such as diabetes and smoking, and a prior history of infection.

A recent analysis of a large US health insurance database identified 5,889 patients treated systemically for psoriasis. ${ }^{45}$ It excluded those who had received solid organ or autologous transplantation, were HIV positive or had advanced liver or kidney disease or cancer. In this real-world setting, the adjusted hazard ratio for hospitalisation and systemic antibiotics for sepsis, pneumonia, skin/soft tissue infection, meningitis, and renal/urinary tract infections was 1.31 (95\% CI, 1.021.68) for biologic vs non- biologic treatment.

\section{Comments/Learning points 2:}

- Psoriasis is independently associated with an increased risk of serious infection.

- The increase in serious infections independently associated with psoriasis appears to consist of common infections such as respiratory, abdominal and skin and soft tissue infections, rather than unusual or opportunistic infections. 
The Australasian Psoriasis registry, based on 11,311 patient years of follow up from 2,170 patients, documented 325 infections (2.87/100 patient years): 44 requiring hospital admission (0.39/100 patient years) and 271 requiring systemic outpatient treatment (2.4/100 patient-years). The highest rate of hospitalised infection was associated with secukinumab, followed by infliximab, ustekinumab, adalimumab and etanercept (personal communication, Australasian Psoriasis Registry, Nov 2017). (1)

There are case reports of infections with bacteria including Nocardia (cutaneous as well as systemic), Legionella, Listeria, Bartonella and Burkholderia pseudomallei (melioidosis). Reactivation of previous unrecognised strongyloides infestation as well as exacerbation of crusted scabies and of leishmaniasis have also been reported. The French RATIO study ${ }^{46}$ recorded 45 serious opportunistic infections: 10 patients required ICU admission and four died. However, reporting bias is inevitable with case reports and small, uncontrolled observational studies, so these need to be interpreted with great care. In general, the risk of serious or unusual infection in patients on systemic treatment for psoriasis remains low and should not be a barrier to offering effective treatment.

All patients starting immune-modulatory treatment should be screened for latent and active tuberculosis. If active disease is present, initiation of methotrexate, ciclosporin or a biologic should be deferred and the patient commenced on antituberculous therapy. It may be considered, on a case-by-case basis, to start the immune-modulator before completion of antituberculous therapy having regard to factors such as compliance with medication, antituberculous drug susceptibility testing, and clinical progress $\quad{ }^{47}$ Acitretin can be used at any stage. Latent tuberculosis is relatively common in certain geographic areas, with rates varying from 5-29\% in patients with psoriasis. ${ }^{48}$ The risk of developing active tuberculosis on an anti-TNF is 5-10 times higher than in patients not exposed to biological therapies, with a mortality of $6-17 \%{ }^{46,49}$ For these reasons, tuberculosis prophylaxis (for example isoniazid, isoniazid/rifapentine, etc.) is recommended for patients with latent tuberculosis, commencing with, or prior to, immune-modulatory treatment. ${ }^{48,50}$

This article is protected by copyright. All rights reserved 


\section{Comments/Learning points 3:}

- All patients starting an immune-modulatory agent should be screened for latent and active tuberculosis. Re-screening should occur when suggested by new clinical findings, including relevant exposures.

- Moderate/high dose systemic corticosteroids may pose a greater risk for reactivation of latent tuberculosis or promotion of other serious infections than current biologics used in psoriasis.

- Avoiding biologic treatment in patients with chronic infection (e.g. TB, HIV, HBV, HCV) should not be automatic but based on individual assessments of the risks and benefits of treatment

A slight increase in candidiasis during treatment with secukinumab, ustekinumab and ixekizumab has been observed, but infections are usually superficial, easily managed with appropriate anti-fungals and do not require cessation of the biologics. ${ }^{11}$ There is an increased risk of herpes zoster during treatment with antiTNF $\alpha$ therapies, which may present atypically as a multi-dermatomal or recurrent infection, complicated by encephalitis and vasculopathy.

Initiation of immune-suppressant therapies should be deferred until resolution of acute hepatitis B virus (HBV) infection. The risk of reactivation of past hepatitis B virus infection from systemic anti-psoriasis therapies is low, but some drugs (e.g. acitretin and methotrexate) may contribute to cumulative liver injury, so should be used with care. The risk of HBV reactivation from biologics, although greater than from systemic therapies, appears to be low. ${ }^{52}$ None-the-less, for inactive carriers of chronic HBV infection commencing TNF $\alpha$-inhibitors, for whom antiviral therapy would not otherwise be indicated, antiviral prophylaxis is recommended (local infectious disease protocols may recommend lamivudine, tenofovir or entecavir); these should be started prior to and continue for 6-12 months longer than the biologic. 53

\section{Comments/Learning points 4:}


- Tuberculosis: Patients with latent tuberculosis should receive prophylactic antituberculous treatment if being commenced on immune-modulatory therapy.

- Hepatitis B: For inactive carriers of hepatitis B, where antiviral therapy is not already indicated, antiviral prophylaxis is recommended for HBV sAg +ve cases, commencing prior to the anti-TNF- $\alpha$ agent and continued for 6-12 months after the anti-TNF- $\alpha$ agent has been stopped.

- Hepatitis C: Once a sustained virologic response (SVR12) has been achieved following treatment of chronic hepatitis $\mathrm{C}$ with direct-acting antiviral therapy, the risk of late relapse is extremely rare.

- HIV: Patients with human immunodeficiency virus can be treated with systemic and biologic therapies, but close monitoring of immunologic status, virologic control, adverse drug reactions and malignancy is essential.

The new direct-acting antiviral therapies are extremely effective in treating hepatitis C virus (HCV) infection, with sustained virologic responses (SVR) of $>90 \%$ in most patient groups with 8-24 week regimens. As late relapse following sustained virologic response is rare, ${ }^{54}$ both systemic and biologic treatments may be considered if the patient has achieved a sustained virologic response (SVR12);55,56 however safety data is limited, so a precautionary approach is to wait for several further months before starting a biologic. Despite cure/clearance of HCV, there may be persisting fibrosis/cirrhosis, so there may be a preference to use either ciclosporin or an anti-TNF $\alpha$ agent, over acitretin or methotrexate, although this is largely based on opinion.

The increased occurrence of comorbid disease amongst people living with HIV underlines the importance of addressing psoriasis in these patients. ${ }^{57}$ Acitretin can be used, although there may be an increased risk of hepatotoxicity. Acitretin is not immunosuppressive. Methotrexate may also be considered, but there are case reports of Pneumocystis carinii pneumonia, cryptococcosis and histoplasmosis, although these are more likely the result of the underlying HIV than methotrexate. 
Ciclosporin may have beneficial effects when used to treat psoriasis that presents during primary HIV infection, or when starting antiretroviral therapy (first 8 weeks of therapy). The immunosuppressive effect of ciclosporin becomes greater with prolonged treatment, leading to the recommendation to limit treatment to 3-6 month cycles of treatment. ${ }^{58}$ Hydroxyurea may also be an option. ${ }^{59}$

Biologics are likely to be effective in the management of HIV patients with severe psoriasis. ${ }^{57}$ Essential requirements for using a biologic include baseline stable antiretroviral therapy with virologic suppression $(<20-50$ copies $/ \mathrm{ml})$, and close clinical monitoring.

Adalimumab has been associated with HTLV-1 reactivation and several cases of adult T-cell leukaemia and lymphoma (ATLL) have been reported. ${ }^{60}$

\section{Comments/Learning points 5 :}

- Whether to, and when to, resume immune-modulatory treatment after an episode of serious infection depends on risk assessment for that patient, taking into account the risk of recurrent infection, any interventions that can modify the risk, and the need for psoriasis control.

- Many serious infections will be cured within a single dosing interval of a biologic drug.

- For infections requiring prolonged treatment, biologics might be carefully resumed during treatment for a serious infection on a case-by-case basis.

\section{General comments}

All serious infections should be treated prior to commencing systemic or biologic therapies in patients with psoriasis. It is inevitable that some patients on systemic anti-psoriasis treatments will develop a serious infection such as malaria, legionella pneumonia, S. aureus bloodstream infection, etc. during treatment. Depending on the severity and seriousness of the infection, its treatment, or sequelae, the antipsoriasis treatments, possibly with the exception of acitretin, should be withheld until the patient has recovered.

This article is protected by copyright. All rights reserved 
There is no standard period of time before any psoriasis treatment should be restarted, but an empiric 2-4 weeks minimum stand down period is reasonable. Issues to consider in deciding whether to restart psoriasis treatment include the risks and benefits for recurrent serious infection, whether there are any modifiable risks for prevention of recurrent infection (e.g., antibiotic prophylaxis, vaccination, avoidance of activities/exposures), the consequence of loss of psoriasis control and the availability of other psoriasis treatment options.

\section{Psoriasis, wound healing and surgery}

Concerns have been expressed over the risk of surgical site infection and wound dehiscence after surgery in patients with psoriasis. The clinical evidence for this is generally of low strength and quality. Studies examining the effect of biologic therapy on perioperative complications are mostly retrospective, uncontrolled, lacking statistical power to determine a difference between continuing versus stopping therapy, and potentially confounded by the clinical reasons for continuing or stopping treatment. Psoriasis relapse does occur with the perioperative cessation of therapy, and concerns have been raised regarding the potential for development of anti-drug antibodies with intermittent administration of biologic drugs, causing reduced treatment efficacy. On the other hand, studies show that wound healing is either normal, or slightly increased in untreated patients with psoriasis. ${ }^{61}$

A recent systematic review of perioperative management of systemic immune modulators in patients with psoriasis and psoriatic arthritis, ${ }^{62}$ based on level III evidence, indicated that infliximab, adalimumab, etanercept, methotrexate and ciclosporin can safely be continued through low-risk operations. For moderate- and high-risk surgeries, a case-by-case approach should be taken based on the characteristics of the patient and the planned surgical procedure. These may include the likelihood and anticipated severity of a psoriasis relapse if therapy were stopped, the consequences of any infection or poor wound healing and the patients' evaluation of the risks of infection vs disease flare ${ }^{63}$. In addition, the level of immune-modulation from systemic therapy including biologic and other patient risk 
factors for infection, such as corticosteroid use, age, smoking, diabetes, nutritional state, should be taken into account.

\section{Comments/Learning points 6:}

- Wound healing in psoriasis is normal.

- Infliximab, adalimumab, etanercept, methotrexate and ciclosporin can be safely continued through low-risk surgical procedures.

- For moderate- and high-risk surgeries, a case-by-case approach should be taken based on the patients' individual risk factors and comorbidities.

As a general indication, if a 'no-treatment' effect is desired,'2 the systemic agent should be stopped 3-5 half-lives, or the length of the treatment cycle (whichever is longer), before planned surgery.

\section{Prevention of infection}

As any patient with moderate to severe psoriasis may progress to immunemodulatory therapies, it is important that their immunisations are up to date. ${ }^{64-67} \mathrm{In}$ the Australia/New Zealand context, ${ }^{64,65}$ ensure recommended immunisations are up to date, including pneumococcal and annual influenza vaccination and any ageappropriate boosters, e.g. for tetanus and pertussis. Vaccination of household contacts, e.g., against influenza, pertussis, varicella, and zoster, should also be considered.

Inactivated vaccines may be administered whilst on systemic therapy (methotrexate, ciclosporin and biologics) although the potential for reduced immunogenicity exists. Post-vaccination serologic testing may be appropriate, with additional doses given if seroconversion hasn't occurred. Live vaccines (e.g. MMR, varicella zoster, yellow fever) are generally contraindicated in immune-suppressed individuals, but this depends on the degree of immune suppression and individual risk factors. ${ }^{64}$ Tuberculosis vaccine (BCG) is always contraindicated.

\section{Comments/Learning points 7:}

This article is protected by copyright. All rights reserved 
- Make sure all psoriasis patient's immunisations are up to date, including annual influenza.

- Live vaccines are generally contraindicated in those receiving systemic immunosuppressive therapy, but this depends on the degree of immune suppression and individual risk factors.

Live vaccines, such as MMR and varicella zoster, can be given to patients receiving low dose systemic therapy (i.e. methotrexate $\leq 0.4 \mathrm{mg} / \mathrm{kg}$ per week, azathioprine $\leq 3.0 \mathrm{mg} / \mathrm{kg}$ per day, mercaptopurine $\leq 1.5 \mathrm{mg} / \mathrm{kg}$ per day), or low-dose corticosteroids ( $<20 \mathrm{mg} /$ day prednisone). The recommendation for biologics is more complex, but most recommend stopping them for at least 6 months before giving live vaccines, and for at least 12 months in the case of live attenuated Herpes zoster vaccine.

Oral typhoid vaccines should be avoided. Use inactivated parenteral typhoid Vi polysaccharide vaccine instead. Yellow fever vaccine is generally contraindicated, but should be considered on a case-by-case basis.

\section{Conclusions}

The advent of specific immune-modulatory psoriasis treatments has highlighted the associations of psoriasis with infection. Whilst the risk of infections is increased with these treatments, it is difficult to tease out the many confounding factors, including severity of psoriasis, smoking, diabetes, metabolic syndrome, etc. It is inevitable that some patients on systemic anti-psoriasis treatments will develop serious infections during treatment, but the absolute risk is low. When to stop immune-modulatory anti-psoriasis treatments depends on the seriousness of the infection and/or its treatment. Issues to consider when re-starting immunemodulatory treatment include the risk of recurrent serious infection in that patient and the consequence of not treating the patient's psoriasis. 


\section{$\underline{\text { References }}$}

1. Rademaker M, Gupta M, Andrews M, et al. The Australasian Psoriasis

Collaboration view on Methotrexate for psoriasis in the Australasian setting. Australas J Dermatol. 2017; 58: 166-70.

2. Rademaker M, Agnew K, Andrews M, et al. Psoriasis in those planning a family, pregnant or breast-feeding. The Australasian Psoriasis Collaboration. Australas J Dermatol. 2017 May 25. doi: 10.1111/ajd.12641.

3. Fahlén A, Engstrand L, Baker BS, Powles A, Fry L. Comparison of bacterial microbiota in skin biopsies from normal and psoriatic skin. Arch Dermatol Res. 2012;304:15-22.

4. Alekseyenko AV, Perez-Perez GI, De Souza A, et al. Community differentiation of the cutaneous microbiota in psoriasis. Microbiome. 2013;1:31.

5. Fry L, Baker BS, Powles AV Fahlen A, Engstrand L. Is chronic plaque psoriasis triggered by microbiota in the skin? Br J Dermatol. 2013;169:47-52.

6. Danese S. New therapies for inflammatory bowel disease: from the bench to the bedside. Gut 2012;61:918-32.

7. Elson CO, Alexander KL. Host-microbiota interactions in the intestine. Dig Dis. 2015;33:131-6.

8. Telfer NR, Chalmers RJ, Whale K, Colman G. The role of streptococcal infection in the initiation of guttate psoriasis. Arch Dermatol. 1992;128:39-42.

9. Gudjonsson JE, Thorarinsson AM, Sigurgeirsson B, Kristinsson KG, Valdimarsson H..Streptococcal throat infections and exacerbation of chronic plaque psoriasis: a prospective study. Br J Dermatol. 2003;149:530-4.

10. Rasmussen JE. The relationship between infection with group A beta hemolytic streptococci and the development of psoriasis. Pediatr Infect Dis . 2000;19:153-4. 
11. Owen CM, Chalmers RJ, O'Sullivan T, Griffiths CE. A systematic review of antistreptococcal interventions for guttate and chronic plaque psoriasis. Br J Dermatol. 2001;145:886-90.

12. Mallon E, Bunce M, Savoie H, et al. HLA-C and guttate psoriasis. Br J Dermatol. 2000;143:1177-82.

13. Sigürdardottir S, Thorleifsdottir RH, Valdimarsson H, Johnston A. The association of sore throat and psoriasis might be explained by histologically distinctive tonsils and increased expression of skin-homing molecules by tonsil T cells. Clin Exp Immunol. 2013;174:139-51.

14. Garritsen FM, Kraag DE, de Graaf M. Guttate psoriasis triggered by perianal streptococcal infection. Clin Exp Dermatol. 2017;42:536-8.

15. Mrowietz U. Pustular eruptions of palms and soles. In: Fitzpatrick TB, Wolff K (eds). Fitzpatrick's Dermatology in General Medicine ), 7th edn, Vol. 1. New York: McGraw-Hill Medical, 2008;215-8.

16. Ng CY, Huang YH, Chu CF, Wu TC, Liu SH.. Risks for Staphylococcus aureus colonization in psoriasis patients: A systematic review and meta-analysis. $\mathrm{Br} \mathrm{J}$ Dermatol. 2017;177:967-7.

17. Tomi NS, Kränke B, Aberer E. Staphylococcal toxins in patients with psoriasis, atopic dermatitis and erythroderma, and in healthy control subjects. J Am Acad Dermatol. 2005;53:67-72.

18. Skinner RB, Rosenberg EW, Noah PW. Antimicrobial treatment of psoriasis. Dermatol Clin. 1995;13:909-13.

19. Rosenberg EW, Skinner RB, Noah PW. Anti-infectious therapy of psoriasis. In: Psoriasis (Roenigk HH, Maibach HI, eds), 3rd edn. New York: Marcel Dekker Inc., 1998:37-9.

This article is protected by copyright. All rights reserved 
20. Onsun N. Arda Ulusal H, Su O, Beycan I, Biyik Ozkaya D, Senocak M. Impact of Helicobacter pylori infection on severity of psoriasis and response to treatment. Eur J Dermatol. 2012;22:117-20.

21. Ungprasert $\mathrm{P}, \mathrm{Wijarnpreecha} \mathrm{K}$, Wetter DA. Periodontitis and risk of psoriasis: a systematic review and meta-analysis. J Eur Acad Dermatol Venereol. 2017;31:85762.

22. Horton DB, Scott FI, Haynes K, et al. Antibiotic Exposure, Infection, and the Development of Pediatric Psoriasis: A Nested Case-Control Study. JAMA Dermatol. 2016;152:191-9.

23. Stinco G, Fabris M, Pasini E, et al. Detection of DNA of Chlamydophila psittaci in subjects with psoriasis: a casual or a causal link? Br J Dermatol. 2012;167:926-8.

24. Taheri Sarvtin M, Shokohi T, Hajheydari Z, Yazdani J, Hedayati MT. Evaluation of candidal colonization and specific humoral responses against Candida albicans in patients with psoriasis. Int J Dermatol. 2014;53:e555-60.

25. Buslau M, Menzel I, Holzmann H. Fungal flora of human faeces in psoriasis and atopic dermatitis. Mycoses. 1990;33:90-4.

26. Waldman A, Gilhar A, Duek L, Berdicevsky I. Incidence of Candida in psoriasis-a study on the fungal flora of psoriatic patients. Mycoses. 2001;44:77-81.

27. Fry L, Baker BS. Triggering psoriasis: the role of infections and medications. Clin Dermatol. 2007;25:606-15.

28. Buslau M, Hänel H, Holzman H. The significance of yeasts in seborrheic dermatitis. Hautarzt 1989;40:611-3.

29. Prohic A, Jovovic Sadikovic T, Krupalija-Fazlic M, Kuskunovic-Vlahovljak S. Malassezia species in healthy skin and in dermatological conditions. Int J Dermatol. 2016;55:494-504.

This article is protected by copyright. All rights reserved 
30. Farr PM, Krause LB, Marks JM, Shuster S. Response of scalp psoriasis to oral ketoconazole. Lancet 1985;2:921-2.

31. Cronin JG, Mesher D, Purdie K, et al. Beta-papillomaviruses and psoriasis: an intra-patient comparison of human papillomavirus carriage in skin and hair. Br J Dermatol. 2008;159:113-9.

$$
\text { - }
$$

32. Molés JP, Hadi JC, Guilhou JJ. High prevalence of an IgG response against murine leukaemia virus (MLV) in patients with psoriasis. Virus Res. 2003;94:97-101.

33. Chen H, Hayashi G, Lai OY, et al. Psoriasis patients are enriched for genetic variants that protect against HIV-1 disease. PLoS Genet 2012;8:e1002514.

34. Duvic M, Johnson TM, Rapini RP, Freese T, Brewton G, Rios A. Acquired immunodeficiency syndrome-associated psoriasis and Reiter's syndrome. Arch Dermatol. 1987;123:1622-32.

35. Yen YF, Jen IA, Chen M, et al. HIV infection increases the risk of incident psoriasis: a nationwide population-based cohort study in Taiwan. J Acquir Immune Defic Syndr. 2017;75:493-9.

36. Cohen AD, Weitzman D, Birkenfeld S, Dreiher J. Psoriasis associated with hepatitis C but not with hepatitis B. Dermatology. 2010;220:218-22.

37. ImafukuS, Nakayama J. Profile of patients with psoriasis associated with hepatitis C virus infection. J Dermatol. 2013;40:428-33.

38. Chun K, Afshar M, Audish D, et al. Hepatitis C may enhance key amplifiers of psoriasis. J Eur Acad Dermatol Venereol. 2017;31:672-8.

39. Seetharam KA, Sridevi K. Chikungunya infection: a new trigger for psoriasis. J Dermatol. 2011;38:1033-4.

40. Wakkee M, de Vries E, van den Haak P, Nijsten T. Increased risk of infectious disease requiring hospitalization among patients with psoriasis: a population-based cohort. J Am Acad Dermatol. 2011;65:1135-44.

This article is protected by copyright. All rights reserved 
41. Takeshita J, Shin DB, Ogdie A, Gelfand JM. Increased risk of serious infection among patients with psoriasis: a population-based cohort study in the United Kingdom. J Invest Dermatol. 2016;136(5 suppl 1):S34.

42. Kao LT, Lee CZ, Liu SP, Tsai MC, Lin HC. Psoriasis and the risk of pneumonia: a population-based study. PLoS ONE. 2014;9:e116077.

43. Garcia-Doval I, Cohen AD, Cazzaniga S, et al. Risk of serious infections, cutaneous bacterial infections, and granulomatous infections in patients with psoriasis treated with anti-tumor necrosis factor agents versus classic therapies: Prospective metaanalysis of Psonet registries. J Am Acad Dermatol. 2017;76:299-308.

44. Kalb RE, Fiorentino DF, Lebwohl MG, et al. Risk of serious infection with biologic and systemic treatment of psoriasis: Results From the Psoriasis Longitudinal Assessment and Registry (PSOLAR). JAMA Dermatol. 2015;151:961-9.

45. Dobry AS, Quesenberry CP, Ray GT, Geier JL, Asgari MM. Serious infections among a large cohort of subjects with systemically treated psoriasis. J Am Acad Dermatol. 2017;77:838-44.

46. Salmon-Ceron D, Tubach F, Lortholary 0, et al. Drug-specific risk of nontuberculosis opportunistic infections in patients receiving anti-TNF therapy reported to the 3-year prospective French RATIO registry. Ann Rheum Dis. 2011;70:616-23.

47. British Thoracic Society Standards of Care Committee. BTS recommendations for assessing risk and for managing Mycobacterium tuberculosis infection and disease in patients due to start anti-TNF-alpha treatment. Thorax. 2005;60:800-5.

48. Gisondi P, Pezzolo E, Lo Cascio G, Girolomoni G. Latent tuberculosis infection in patients with chronic plaque psoriasis who are candidates for biological therapy. $\mathrm{Br}$ J Dermatol. 2014;171:884-90.

49. Dixon WG, Hyrich KL, Watson KD, et al. Drug-specific risk of tuberculosis in patients with rheumatoid arthritis treated with anti-TNF therapy: results from the

This article is protected by copyright. All rights reserved 
British Society for Rheumatology Biologics Register (BSRBR). Ann Rheum Dis. 2010; 69: 522-8.

50. Conti A, Piaserico S, Gisondi P, et al. Management of long-term therapy with biological drugs in psoriatic patients with latent tuberculosis infection in real life setting. Dermatol Ther. 2017;30. doi: 10.1111/dth.12503. Epub 2017 May 26.

51. Saunte DM, Mrowietz U, Puig L, Zachariae C. Candida infections in patients with psoriasis and psoriatic arthritis treated with interleukin-17 inhibitors and their practical management. Br J Dermatol. 2017;177:47-62

52. Bessone F, Dirchwolf M. Management of hepatitis B reactivation in immunosuppressed patients: an update on current recommendations. World J Hepatol. 2016;8:385-94.

53. Cannizzaro MV, Franceschini C, Esposito M, Bianchi L, Giunta A. Hepatitis B reactivation in psoriasis patients treated with anti-TNF agents: prevention and management. Psoriasis (Auckl). 2017;7:35-40.

54. Simmons B, Saleem J, Hill A, Riley RD, Cooke GS. Risk of Late Relapse or Reinfection With Hepatitis C Virus After Achieving a Sustained Virological Response: A Systematic Review and Meta-analysis. Clin Infect Dis. 2016;62:683-94.

55. Hahn KJ, Kohli A, Sims Z, Kottilil S. Durable Sustained Virologic Response After Oral Directly Acting Antiviral Therapy Despite Immunosuppressive Treatment. Open Forum Infect Dis. 2015;2(3):ofv091.

56. Bonifati C, Lora V, Graceffa D, Nosotti L. Management of psoriasis patients with hepatitis B or hepatitis C virus infection. World J Gastroenterol. 2016;22:6444-55

57. Nakamura M, Abrouk M, Farahnik B, Zhu TH, Bhutani T. Psoriasis treatment in HIV-positive patients: a systematic review of systemic immunosuppressive therapies. Cutis. 2018;101:38;42;56.

58. Muellenhoff MW, Koo JY. Cyclosporine and skin cancer: an international dermatologic perspective over 25 years of experience. A comprehensive review and This article is protected by copyright. All rights reserved 
pursuit to define safe use of cyclosporine in dermatology. J Dermatolog Treat. 2012;23:290-304

59. Lee ES, Heller MM, Kamangar F, Park K, Liao W, Koo J. Hydroxyurea for the Treatment of Psoriasis including in HIV-infected Individuals: A Review. Psoriasis Forum. 2011;17:180-7

60. Bittencourt AL, Oliveira PD, Bittencourt VG, Carvalho EM, Farre L. Adult T-cell leukemia/lymphoma triggered by adalimumab. J Clin Virol. 2013;58:494-6.

61. Morhenn VB, Nelson TE, Gruol DL. The rate of wound healing is increased in psoriasis.J Dermatol Sci. 2013;72:87-92.

62. Choi YM, Debbaneh M, Weinberg JM, et al. From the Medical Board of the National Psoriasis Foundation: Perioperative management of systemic immunomodulatory agents in patients with psoriasis and psoriatic arthritis. J Am Acad Dermatol. 2016;75:798-805.

63. Goodman SM, Miller AS, Turgunbaev M, et al. Clinical Practice Guidelines: Incorporating Input From a Patient Panel. Arthritis Care Res (Hoboken). 2017;69:1125-1130.

64. The Australian Immunisation Handbook 10th Edition. http://immunise.health.gov.au/internet/immunise/publishing.nsf/Content/Handb ook10-home handbook10part3 handbook10-3-3. Accessed 5.02.2018.

65. Ministry of Health. 2018. Immunisation Handbook 2017 (2nd edn). Wellington: Ministry of Health.

66. Smith CH, Jabbar-Lopez ZK, Yiu ZZ, et al. British Association of Dermatologists guidelines for biologic therapy for psoriasis 2017. Br J Dermatol. 2017;177:628-36.

67. Wine-Lee L, Keller SC, Wilck MB, Gluckman SJ, Van Voorhees AS. From the Medical Board of the National Psoriasis Foundation: Vaccination in adult patients on systemic therapy for psoriasis. J Am Acad Dermatol. 2013;69:1003-13. 


\section{University Library}

\section{- M I N E R VA}

\section{A gateway to Melbourne's research publications}

Minerva Access is the Institutional Repository of The University of Melbourne

\section{Author/s:}

Rademaker, M;Agnew, K;Anagnostou, N;Andrews, M;Armour, K;Baker, C;Foley, P;Gebauer, K;Gupta, M;Marshman, G;Rubel, D;Sullivan, J;Wong, L-C

Title:

Psoriasis and infection. A clinical practice narrative

Date:

2019-05-01

\section{Citation:}

Rademaker, M., Agnew, K., Anagnostou, N., Andrews, M., Armour, K., Baker, C., Foley, P., Gebauer, K., Gupta, M., Marshman, G., Rubel, D., Sullivan, J. \& Wong, L. -C. (2019). Psoriasis and infection. A clinical practice narrative. AUSTRALASIAN JOURNAL OF DERMATOLOGY, 60 (2), pp.91-98. https://doi.org/10.1111/ajd.12895.

Persistent Link:

http://hdl.handle.net/11343/284492 